\title{
Human liver-kidney model elucidates the mechanisms of aristolochic acid nephrotoxicity
}

Shih-Yu Chang, ${ }^{1}$ Elijah J. Weber, ${ }^{2}$ Viktoriya S. Sidorenko, ${ }^{3}$ Alenka Chapron, ${ }^{2}$ Catherine K. Yeung, ${ }^{2,4}$ Chunying Gao, ${ }^{2}$ Qingcheng Mao, ${ }^{2}$ Danny Shen, ${ }^{2}$ Joanne Wang, ${ }^{2}$ Thomas A. Rosenquist, ${ }^{3}$ Kathleen G. Dickman, ${ }^{3}$ Thomas Neumann, ${ }^{5}$ Arthur P. Grollman,, ${ }^{3,6}$ Edward J. Kelly, ${ }^{2}$ Jonathan Himmelfarb, ${ }^{4}$ and David L. Eaton ${ }^{1}$

'Department of Environmental and Occupational Health Sciences and 'Department of Pharmaceutics, University of Washington, Seattle, Washington, USA. ${ }^{3}$ Department of Pharmacological Sciences, Stony Brook University, Stony Brook, New York, USA. ${ }^{4}$ Department of Medicine, Division of Nephrology, Kidney Research Institute, University of Washington, Seattle, Washington, USA. ${ }^{5}$ Nortis, Washington, USA. ${ }^{5}$ Department of Medicine, Stony Brook University, Stony Brook, New York, USA.

Environmental exposures pose a significant threat to human health. However, it is often difficult to study toxicological mechanisms in human subjects due to ethical concerns. Plant-derived aristolochic acids are among the most potent nephrotoxins and carcinogens discovered to date, yet the mechanism of bioactivation in humans remains poorly understood. Microphysiological systems (organs-on-chips) provide an approach to examining the complex, species-specific toxicological effects of pharmaceutical and environmental chemicals using human cells. We microfluidically linked a kidney-on-a-chip with a liver-on-a-chip to determine the mechanisms of bioactivation and transport of aristolochic acid I (AA-I), an established nephrotoxin and human carcinogen. We demonstrate that human hepatocyte-specific metabolism of AA-I substantially increases its cytotoxicity toward human kidney proximal tubular epithelial cells, including formation of aristolactam adducts and release of kidney injury biomarkers. Hepatic biotransformation of AA-I to a nephrotoxic metabolite involves nitroreduction, followed by sulfate conjugation. Here, we identify, in a human tissue-based system, that the sulfate conjugate of the hepatic NQO1-generated aristolactam product of AA-I (AL-I-NOSO$)_{3}$ ) is the nephrotoxic form of AA-I. This conjugate can be transported out of liver via MRP membrane transporters and then actively transported into kidney tissue via one or more organic anionic membrane transporters. This integrated microphysiological system provides an ex vivo approach for investigating organ-organ interactions, whereby the metabolism of a drug or other xenobiotic by one tissue may influence its toxicity toward another, and represents an experimental approach for studying chemical toxicity related to environmental and other toxic exposures.

Conflict of interest: T. Neumann, President and CEO of Nortis Inc., has a financial interest in this work, as devices from this company were utilized during the development of these "organ-on-achip" devices.

Submitted: July 6, 2017

Accepted: October 11, 2017

Published: November 16, 2017

Reference information:

JCI Insight. 2017;2(22):e95978.

https://doi.org/10.1172/jci.

insight.95978

\section{Introduction}

Aristolochic acids (AA), produced by plants of the Aristolochiaceae family, have been used worldwide for medicinal purposes for more than 2,500 years, and this practice is still common in many cultures today. $\mathrm{AA}$ are the etiologic agents in the clinical syndromes known as Chinese herb nephropathy and Balkan endemic nephropathy, and they contribute to the global burden of chronic kidney disease and urothelial cancer $(1,2)$. In China alone, 100 million people have taken Aristolochia herbal remedies, highlighting the potential morbidity and mortality associated with medicinal use of these herbs. AA I (AA-I) is now recognized as a potent nephrotoxin that requires bioactivation to a reactive metabolite that forms DNA adducts, including 7-(deoxyadenosin-N6-yl) aristolactam I (dA-AL-I) and 7-(deoxyguanosin-N2-yl) aristolactam I (dG-AL-I) (3). Aristolactam-DNA (AL-DNA) adducts in human tissues serve as biomarkers for AA-I exposure and play a critical role in the development of upper tract urothelial cancers $(4,5)$.

Although numerous in vitro and in vivo animal models have been used in the investigation of biochemical pathways and mechanisms of AL-DNA adduct formation and nephrotoxicity, the pathways 
responsible for AA-I bioactivation in vivo remain a matter of debate, as data obtained from different experimental systems revealed inconsistent results regarding the involvement of sulphotransferases and nitroreductases in AA-I metabolism. In addition to cytochromes P450 (CYPs), other important biotransformation enzymes, including nitroreductases and sulfotransferases (SULTs), are expressed in both liver and kidney $(6,7)$. Thus, the precise toxicological mechanisms of hepatic and renal enzymes, particularly the role of SULTs, to activate or detoxify AA-I in humans remain unclear.

To clarify these toxicological mechanisms and to identify organ-organ interactions that might occur with human tissues in vivo, we utilized an approach involving microfluidic organs-on-chips or "microphysiological systems" (MPS). Such approaches have the advantage of utilizing human-derived cell/tissue cultures in continuously perfused chambers designed to simulate organ and tissue function and response to injury (8). Thus, to evaluate the role of hepatic metabolism in AA-induced kidney injury, we linked a human kidney-on-a-chip (MPS) that recapitulates the renal proximal tubule (9) to a MPS populated with human hepatocytes (Figure 1).

This is the first study to our knowledge to show that microfluidically linked organs-on-chips can be used to identify organ-organ interactions in response to chemical toxicants, combining expertise in synthetic and analytical chemistry, molecular, and cell biology.

\section{Results}

Hepatic metabolism bioactivates AA-I toxicity in human MPS models. We first examined whether hepatic metabolism alters the nephrotoxicity induced by AA-I. Cytotoxicity assays demonstrated that AA-I treatment resulted in greater kidney cell death following prehepatic metabolism (liver $\rightarrow$ kidney) when compared with direct infusion of AA-I into the kidney MPS, indicating that the overall effect of hepatic metabolism is, surprisingly, to bioactivate, rather than to detoxify, AA-I (Figure 2, A and B; methods in Supplemental Figure 1; $P<0.05$; supplemental material available online with this article; https://doi.org/10.1172/jci. insight.95978DS1).

Immunocytochemical staining of DNA and protein AL adducts in the kidney MPS (Figure 2C; negative control, Supplemental Figure 2) revealed more AL adducts following prehepatic metabolism than direct exposure to AA-I. The concentration of kidney injury molecule-1 (KIM-1), a biomarker of kidney toxicity (10), was increased in effluents of kidney MPS following prehepatic AA-I metabolism for 24 hours (Figure 2D, 2.5-fold compared with the vehicle control at $56.4 \pm 13.7 \mathrm{pg} / \mathrm{ml} ; 3.2$-fold compared with the kidney-only group at $25 \mu \mathrm{M}$ with $43.3 \pm 12.2 \mathrm{pg} / \mathrm{ml} ; P<0.01)$. AA-I bioactivation by hepatic metabolism was also found using rat, rather than human, cells (Supplemental Figure 3). Collectively, our results demonstrate that hepatic biotransformation of AA-I results in an approximately 5 -fold net increase in toxicity to both human and rat proximal tubules when compared with direct proximal tubule exposure.

Cotreatment of liver and kidney cells with the NQO1 inhibitor, dicumarol, attenuates AA-I nephrotoxicity. We cotreated cells in the MPS system with both AA-I and the nitroreductase inhibitor, dicumarol, to test the hypothesis that nitroreductase activity is involved in AA-I activation. NADPH:quinone oxidoreductase 1 (NQO1) has previously been implicated in AA reduction to the reactive lactam (11). However, basal expression of NQO1 is relatively low in normal hepatic tissues but can be induced by liver injury (12) and is known to be stimulated by AA-I ingestion in mice (13). Accordingly, we found that AA-I exposure induced NQO1 expression in human liver MPS (Supplemental Figure 4). Coincubation of hepatocytes with $10 \mu \mathrm{M}$ dicumarol attenuated AA-I-induced nephrotoxicity by 39\% (Figure 3, $P<0.01$ ). Although not statistically significant, dicumarol tended to decrease AA-I toxicity after direct infusion into the kidney MPS $(P=0.21)$, suggesting a minor role of NQO1 for direct activation of AA-I in the kidney.

Our results indicate that NQO1 in human hepatocytes is important for AA-I nephrotoxicity, through the formation of N-hydroxyaristolactam I (AL-I-NOH). It remains possible that other nitroreductases, such as cytochrome $\mathrm{P} 450$ oxidoreductase and/or xanthine dehydrogenase could also be involved in the hepatic activation of AA-I via nitroreduction.

Sulfate-conjugated AL-I metabolite is directly toxic to human kidney cells, and this is not modified by hepatic metabolism. Studies on the involvement of phase II metabolism in further bioactivation of AL-I-NOH via a sulfonation step in vivo have revealed conflicting results in different model systems $(6,7,14-16)$. To assess which metabolite of AA-I is the toxic form transported from liver to kidney, human kidney MPS were treated with synthetic sulfate-conjugated AL-I metabolite $\left(\mathrm{AL}-\mathrm{I}-\mathrm{NOSO}_{3}\right)$ or AL-I-NOH. AL-I-NOSO ${ }_{3}$ treatment resulted in 
A

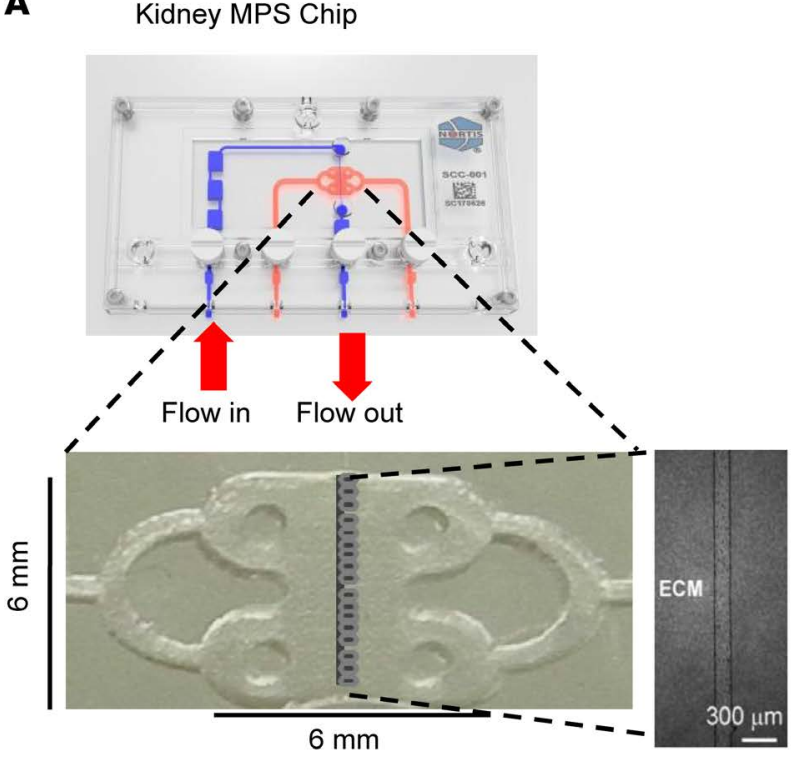

B

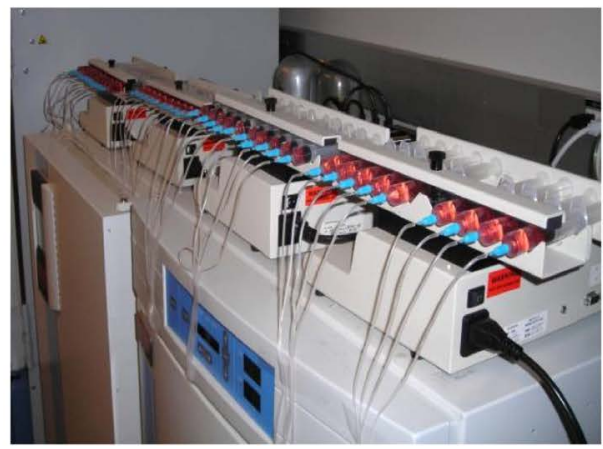

Liver MPS Chip

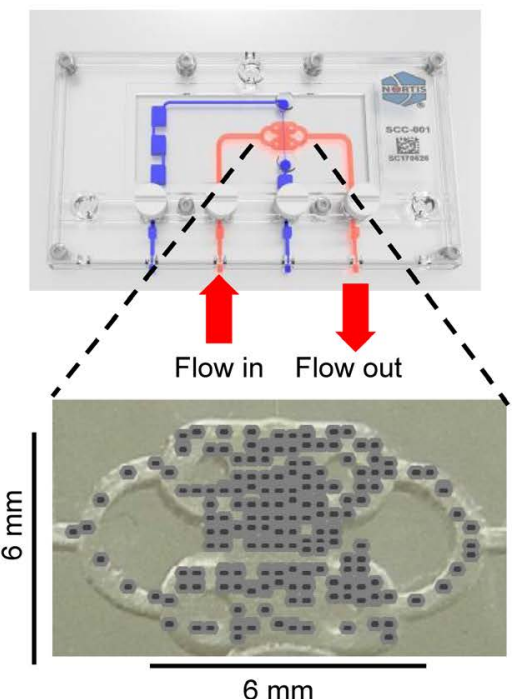

C

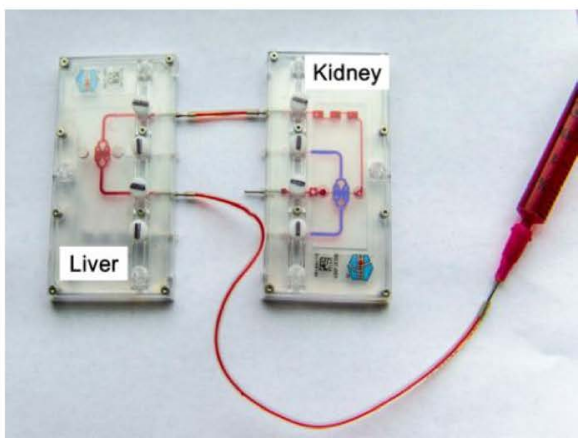

Figure 1. Integrated liver-kidney microphysiological system model. (A) Individual liver and kidney microphysiological system (MPS) chips. Proximal tubular epithelial cells were injected into MPS filled with type I collagen $(6 \mathrm{mg} / \mathrm{ml})$ to form tubular structure as previously described (9, 43). Hepatocytes were seeded into the whole chamber with Matrigel $(0.23 \mathrm{mg} / \mathrm{ml})$ overlay. Liver MPS and kidney MPS were separately maintained, as described in the Methods. Gray dots represent cells. (B) Syringe pump-mediated flow of culture media through MPS chips. (C) Serial integration of liver and kidney MPS chips by direct coupling with C-Flex tubing.

a similar degree of cytotoxicity in the kidney MPS with and without prehepatic metabolism (Figure 4, A and B), whereas AL-I-NOH lacked nephrotoxic properties in either setting (Supplemental Figure 5). AL-I-NOSO also induced similar levels of AL adduct in the kidney MPS at $10 \mu \mathrm{M} \mathrm{AL-I-NOSO}{ }_{3}$ (Figure 4C), with and without prehepatic metabolism.

These results implicate AL-I-NOSO${ }_{3}$, formed via hepatic conjugation of AL-I-NOH by one or more hepatic SULTs, as the dominant nephrotoxic metabolite of AA-I.

The OAT inhibitor probenecid attenuates $\mathrm{AL}-\mathrm{I}-\mathrm{NOSO}_{3}$ toxicity. Because $\mathrm{AL}-\mathrm{I}-\mathrm{NOSO}_{3}$ is water soluble and unable to diffuse across cell membranes, it is likely that facilitated membrane transport is necessary for nephrotoxicity. Previous animal and in vitro studies suggested that organic anion transporters (OATs) are involved in the site-selective toxicity and renal elimination of AA-I $(17,18)$. To test this hypothesis in a human MPS model, we designed experiments to determine whether membrane transport is involved in the renal uptake of AL-I-NOSO ${ }_{3}$. Human kidney cells in an MPS were treated for 24 hours with $\mathrm{AL}-\mathrm{I}-\mathrm{NOSO}_{3}$ (with and without prehepatic metabolism) and the OAT inhibitor, probenecid. Cotreatment with $2 \mathrm{mM}$ probenecid attenuated the cytotoxicity induced by $25 \mu \mathrm{M}$ AL-I-NOSO in human kidney cells by 50\%-60\% (Figure $5, \mathrm{~A}$ and B, $51.5 \%$ in the kidney-only group and $63 \%$ in the liver $\rightarrow$ kidney group), while $2 \mathrm{mM}$ probenecid alone did not cause significant toxicity in kidney cells (data not shown). 
A

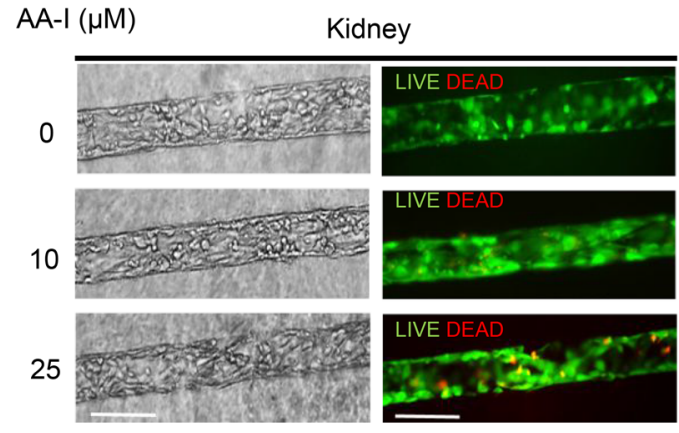

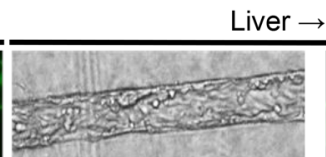
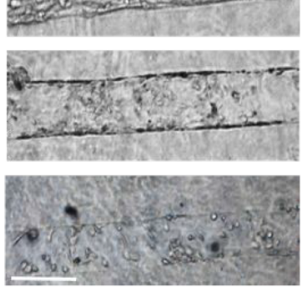

B

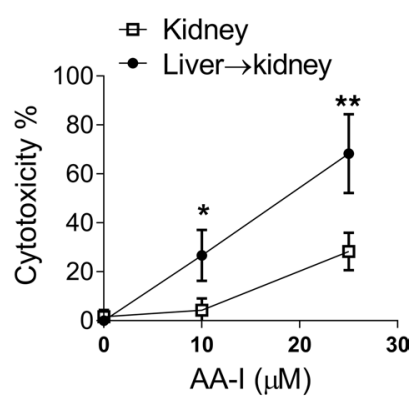

C

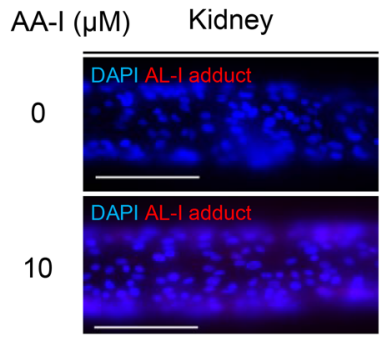

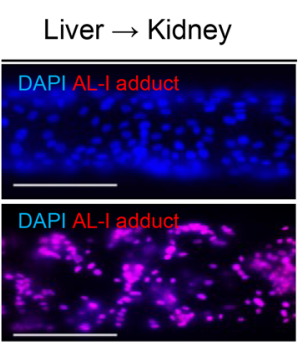

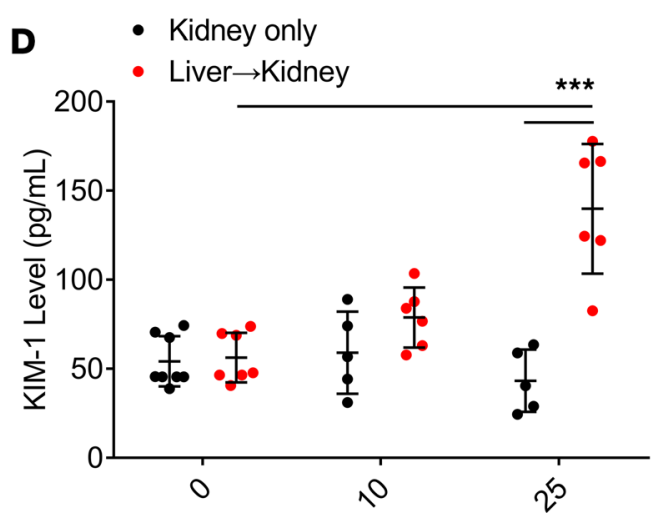

AA-I Conc $(\mu \mathrm{M})$

Figure 2. AA-I human nephrotoxicity with or without hepatic metabolism. (A) Representative phase contrast and LIVE/DEAD images of human proximal tubular epithelial cells (PTECs) cultured in MPS after AA-I treatment for 24 hours. (B) Quantitative cytotoxicity of images in A. $n=5-8$ per treatment group. (C) Representative immunocytochemistry staining of AL-I adducts in 0 and $10 \mu \mathrm{M} A \mathrm{~A}$-I-treated PTECs with or without hepatic metabolism. $n=3$. (D) KIM-1 levels in human MPS effluents. $n=5-8$ per treatment group. All experiments were independently repeated at least 3 times. Scale bars: $150 \mu \mathrm{m}$. Statistical significance was calculated using $t$ test corrected with multiple comparisons. ${ }^{*} P<0.05,{ }^{* *} P<0.01,{ }^{* * *} P<0.001$. Graphs show mean \pm SD.

Since the human kidney MPS recapitulates the polarity of the proximal tubule (9), apical OAT4 is the likely candidate for sulfoconjugate uptake, as it shares similar kinetics to such basolateral transporters of sulfoconjugates expressed in the kidney as OATP4C1 and OAT3 (19).

Additional studies with OAT4-transfected reporter cells confirmed that AL-I-NOSO is a substrate for the OAT4 transporter (Figure 5C). OAT1/3 was also found to transport AL-I-NOSO (Figure 5D).

$A L-I-N_{3} S_{3}$ is likely transported into blood from liver hepatocytes by hepatic membrane transport proteins MRP3 and MRP4. To understand how AL-I-NOSO ${ }_{3}$ gets into blood from the liver, we tested the hypothesis that AL-I-NOSO $\mathrm{N}_{3}$ is transported into blood from the liver by the ABC efflux transporters MRP3 and MRP4. MRP3 and MRP4 were expressed on the basolateral membrane of liver cells using inside-out plasma membrane vesicles. We first verified if MRP3 and MRP4 mediate ATP-dependent uptake of the known MRP substrate, estradiol-17- $\beta$-glucuronide, into membrane vesicles. Uptake of estradiol-17- $\beta$-glucuronide at $10 \mu \mathrm{M}$ into the MRP3 and MRP4 membrane vesicles in the presence of ATP was 50 and 8 times greater, respectively, than that in the presence of AMP (data not shown), demonstrating that MRP3 and MRP4 in the membrane vesicle preparations are functional. Uptake of AL-I-NOSO ${ }_{3}$ at $0.5 \mu \mathrm{M}$ into the MRP3 and MRP4 membrane vesicles was 2.6 and 3.9 times greater, respectively, in the presence of ATP than that in the presence of AMP (Figure 6). The ATP-dependent uptake of AL-I-NOSO 3 was completely abrogated by the highly selective MRP inhibitor MK571. The results demonstrate that AL-I-NOSO is a substrate of both MRP3 and MRP4 for hepatic efflux.

\section{Discussion}

Studies in mice and rats, designed to reproduce AA nephropathy in humans, showed remarkable interstrain and species-to-species differences in renal toxicity for AA-I (20-22). These observations are in 

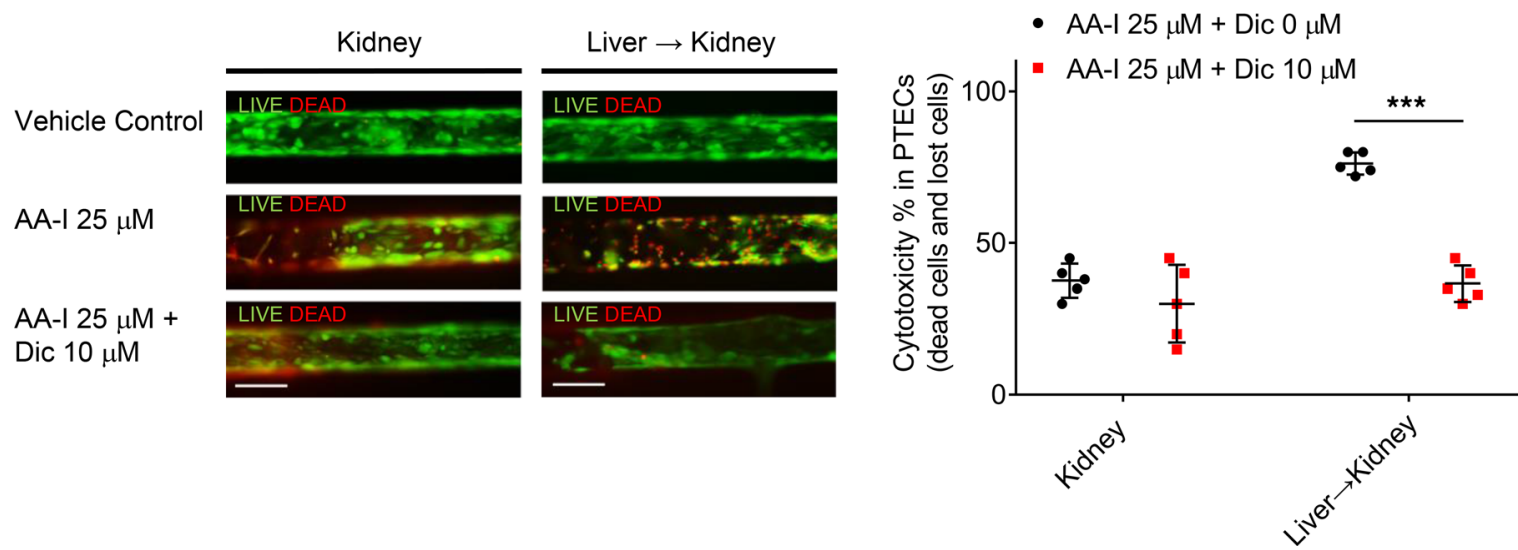

Figure 3. Attenuation of AA-I nephrotoxicity by dicumarol. Representative LIVE/DEAD staining of human proximal tubular epithelial cells cultured in MPS after dicumarol $(10 \mu \mathrm{M})$ and AA-I $(25 \mu \mathrm{M})$ cotreatment for 24 hours. Scale bars: $150 \mu \mathrm{m}$. Quantitative results are presented as average \pm SD. $n=5$ per treatment. Statistical significance was calculated using $t$ test. ${ }^{* *} P<0.001$.

accord with findings in human populations, where only a small fraction of AA-exposed individuals develop renal toxicity and/or urothelial cancer $(4,23,24)$. The genetic basis for such relative susceptibility is yet to be determined.

To set the stage for studies with human integrated liver-kidney organs-on-a-chip, we evaluated AA-I renal toxicity in MPS populated with cells obtained from Sprague-Dawley rats. With respect to the dosage required to elicit tubular damage, rats are the least sensitive rodent species (21). This has been attributed, in part, to the proficiency of isolated rat kidneys perfused with AA-I in producing the primary detoxication products of AA-I, namely, AA-Ia and its O6-conjugates (25). Murine kidneys lack this detoxication pathway. Nevertheless, renal toxicity and forestomach cancers are prominent effects of AA exposure observed in all species of rats and mice tested to date (21).

Based on our results using this integrated MPS model, we conclude that overall biotransformation of AA-I by liver enzymes increases, rather than decreases, toxic effects of AA-I toward kidney cells, as indicated by an increase in cytotoxicity, increased formation of AL adducts, and an increase in the release of the organ-specific injury biomarker, KIM-1 (10), as well as alanine aminotransferase (ALT) (26). To confirm that the renal toxicity occurs due to AA-I metabolites but not proapoptotic factors produced by the liver, we compared the formation of AL-I-DNA and AL-I-protein in kidney-on-a-chip in the presence and absence of liver-on-a-chip. In integrated liver-kidney MPS, the levels of adducted AL-I in renal cells were dramatically higher than those in the kidney MPS alone. Likewise, exposure of the kidney MPS to sulfated metabolite of AA-I induced AL-I adduction in renal cells. These observation suggest that the formation of active metabolites of AA-I in the liver increases its genotoxicities toward the kidney. We then demonstrated that the initial step in the metabolic pathway by which AA-I is converted to toxic metabolites involves nitroreduction (Figure 7). Although several nitroreductases have this potential, NQO1 is considered the most important cytosolic nitroreductase required for AA-I activation (11). Dicumarol, an NQO1 inhibitor (27), reduces AA-I nephrotoxicity and AL-I formation in mice (28), while in rats it promotes AA-I genotoxicity presumably through the inhibition of its detoxification mediated by CYP2C activity in hepatic tissue (29). Once AA-I has been reduced by NQO1 or other nitroreductase(s), the resulting N-hydroxyaristolactam I (AL-I-NOH) is the precursor for reactive cyclic nitrenium/carbenium species and AL-I-DNA adducts (30). Previous work has suggested that this metabolite may directly lead to the formation of active species or undergo sulfonation catalyzed by SULTs (Figure 7) $(6,7)$.

A requirement for phase II metabolism in AA-I metabolism to produce a directly nephrotoxic chemical species in vivo was previously uncertain. Here, we demonstrate conclusively that SULT involvement is required for complete AA-I bioactivation because direct exposure of human kidney cells to AL-I-NO$\mathrm{SO}_{3}$ caused severe cytotoxicity in the absence of prehepatic metabolism (kidney-only group), an effect not observed with AL-I-NOH. Furthermore, the organic anionic transporter inhibitor probenecid inhibited the toxicity of $\mathrm{AL}-\mathrm{I}-\mathrm{NOSO}_{3}$ in human kidney MPS, supporting the hypothesis that formation and subsequent active transport of the sulfate conjugate into proximal tubular epithelial cells (PTECs) is necessary for AA-I nephrotoxicity via the uptake of OATs, particularly OAT1, OAT3, and OAT4 (Figure 7). 
A

AL-I-NOSO

10

25

C

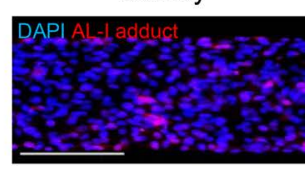

Kidney

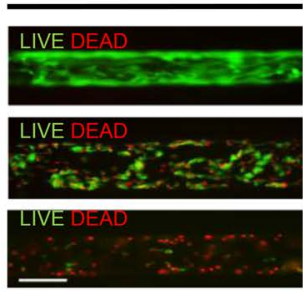

Kidney

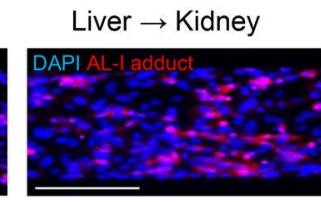

B

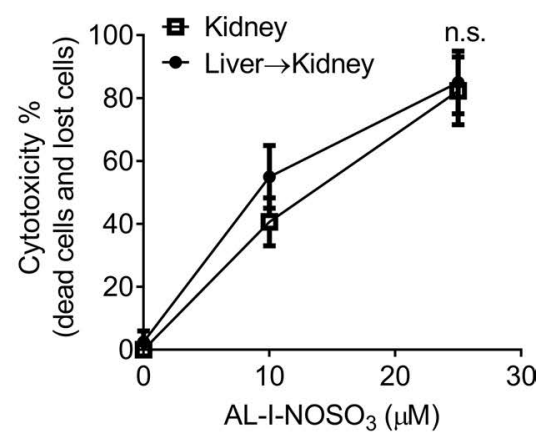

Figure 4. AL-I-NOSO epithelial cells (PTECs) cultured in MPS after AL-I-NOSO treatment for 24 hours. (B) Quantitative results of images in $\mathbf{A}$. $n=5-8$ per treatment group. Statistical significance was calculated using $t$ test (n.s., $P=0.769$ ). Graph shows mean $\pm \mathrm{SD}$. (C) Representative immunocytochemistry results of $A L-I$ adducts in $10 \mu \mathrm{M} \mathrm{AL-I-NOSO}{ }_{3}$-treated PTECs in MPS without or with hepatic metabolism. $n=3$. Scale bars: $150 \mu \mathrm{m}$.

The observed lack of renal toxicity of AL-I-NOH is in accord with our unpublished observations. Thus, AL-I-NOH toxicity varies substantially depending on experimental systems and cell lines under investigation (V.S. Sidorenko et al., unpublished observations). The absence of its toxic effects may occur due to (a) lack of expression or appropriate isoforms of SULTs and/or (b) deficiency of transport of this compound in cells. Thus, in murine kidney cortical slices, incubation with AL-I-NOH induces less AL-I-DNA in comparison to AL-I-NOSO ${ }_{3}$ and AA-I (K.G. Dickman and V.S. Sidorenko, unpublished observations). Moreover, in kidney cortical slices incubated with $\mathrm{H}^{3}$-AA-I, AL-I-NOH competes for uptake with cold AA-I less efficiently than AL-I-NOSO${ }_{3}$, although the observed difference was modest. Similarly, in mice treated with AL-I-NOH in vivo, only minor renal and hepatic AL-I-DNA adduction was found (V.S. Sidorenko and T.A. Rosenquist, unpublished observations). As AL-I-NOH is an excellent substrate for murine and human SULT (6), the absence of renal toxicities of AL-I-NOH in human MPS is most likely due to the lack of its transport in liver and kidney cells.

Sulfate conjugation of the hydroxylamine is electron withdrawing and the product may be cleaved via heterolytic processes, leading to highly reactive electrophiles that bind covalently to DNA and are mutagenic and carcinogenic. Accordingly, sulfonyloxyaristolactam (AL-I-NOSO$)_{3}$, a product of the sulfonation of AL-I-NOH, is an unstable compound with a half-life of approximately 40 minutes in aqueous media, which readily forms AL-DNA adducts upon incubation with DNA. Several lines of evidence support human SULT1A1 and SULT1B1 as being crucial for activation of AA-I and AL-I-NOH $(6,7,15)$. SULTs are abundantly expressed in the liver but are found only at low levels in the kidney (31). In support of this concept, one of us recently demonstrated that murine hepatic cytosols are much more proficient than comparable renal fractions in converting AL-I-NOH into AL-I-NOSO ${ }_{3}$ and AL-I-DNA (6).

Although hepatic metabolism greatly accentuates AA-I nephrotoxicity, at high concentrations AA-I is still directly toxic to kidney cells, and, thus, the kidney can also activate AA-I conversion to toxic metabolites. Cells in the normal renal cortex express high levels of NQO1 (28). However, attenuation of renal NQO1 activity by dicumarol only slightly decreased AA-I toxicity in kidney MPS, suggesting that enzymes other than NQO1 may participate in AA-I activation in kidney cells. Accordingly, AL-I DNA adduct levels in AA-I-exposed human cell lines with genetic knockdown of NQO1 remained unchanged, compared with cells expressing normal NQO1 levels (7). Other nitroreductases such as NADPH:CYP reductase (POR), prostaglandin $\mathrm{H}$ synthase and xanthine oxidase (XDH) could also potentially be involved in the activation of AA-I via nitroreduction in the kidney (11). Thus, our results suggest that NQO1-mediated nitroreduction occurring in hepatic cells is likely to be the most important pathway for AA-I bioactivation in vivo. In addition, these findings corroborate an early report on AL-I, rather than AA-Ia and AL-Ia, detection in humans (32), suggesting that in human liver nitroreduction of AA-I is more efficient than CYP-mediated oxidation of AA-I into nontoxic metabolites. 

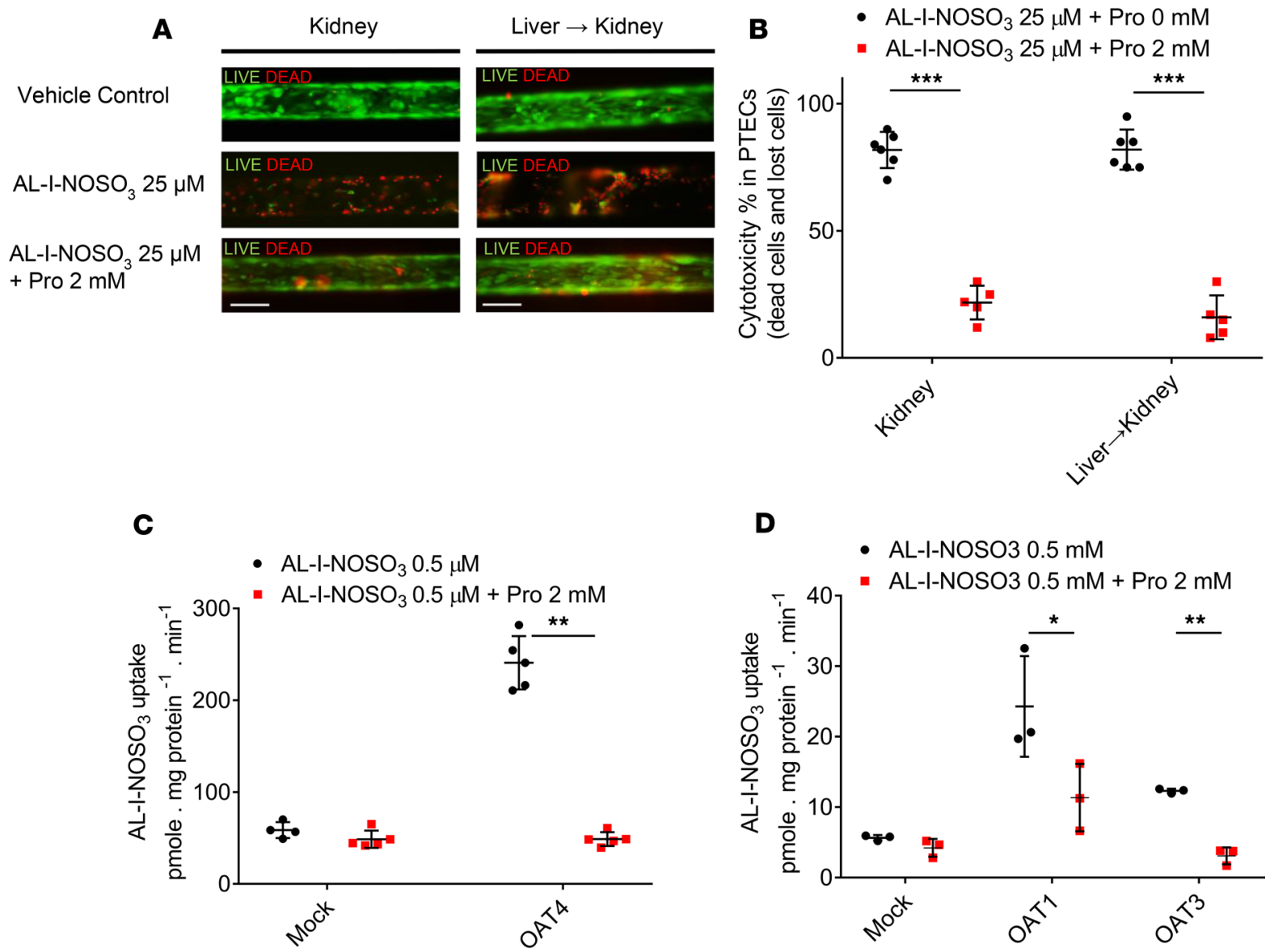

Figure 5. Inhibition of AL-I-NOSO nephrotoxicity by probenecid (OAT inhibitor). (A) Representative LIVE/DEAD staining of human proximal tubular epithelial cells cultured in MPS after probenecid (Pro, $2 \mathrm{mM}$ ) and AL-I-NOSO $(25 \mu \mathrm{M})$ cotreatment for 24 hours. Scale bars: $150 \mu \mathrm{m}$. (B) Quantitative nephrotoxicity of images in A. $n=5-6$ per treatment. (C) Uptake of $0.5 \mu \mathrm{M} \mathrm{AL-I-NOSO}$ in OAT4-transfected COS7 cells versus mock transfection in the presence

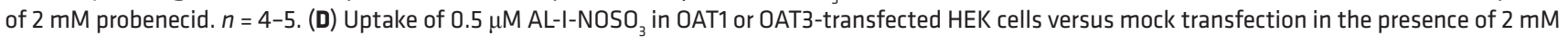
probenecid. $n=3$. Statistical significance was calculated using $t$ test. ${ }^{*} P<0.05,{ }^{* *} P<0.01,{ }^{* *} P<0.001$. Quantitative results are presented as average \pm SD.

Conjugation is generally the final step of biotransformation of xenobiotics necessary to convert lipophilic xenobiotics into water-soluble metabolites that can be excreted in urine or bile, and it is traditionally associated with inactivation or detoxification. However, not all conjugation reactions result in fewer toxic products. For example, SULTs are known to enhance genotoxic effects of polycyclic aromatic hydrocarbons and N-hydroxylamines through the formation of sulfuric acid conjugates (reviewed in refs. 33, 34). These metabolites are chemically unstable and readily undergo solvolysis to produce reactive species (Figure 7).

Bioactivation of xenobiotics by sulfonation in the liver with subsequent delivery of preactivated species to other organs has been proposed for various carcinogens, including 4-aminobiphenyl and 1-methylpyrene (35-37), but the direct evidence on the existence of this pathway in humans is lacking due to the lack of technology and/or instability of active metabolites. Integrated organs-on-chips are perfectly suited to test this hypothesis. Our results provide what we believe to be the first evidence that, in human liver tissue, AA-I may be activated and thus converted to AL-I-NOSO ${ }_{3}$, which is then actively transported out of the liver. MRP3 and MRP4 are ATP-binding cassette efflux transporters localized in the basolateral membrane of liver hepatocytes and are known to transport organic anion conjugates (reviewed in ref. 38). We demonstrated that MRP3 and MRP4 are likely the transporters that mediate efflux of AL-I-NOSO from hepatocytes into the blood. However, other efflux transporters, such as MRP6, cannot be excluded, as we also found some AL-I-NOSO 3 transport activity in control membrane vesicles with no MRP3 or MRP4 overexpression, and this activity could be inhibited by the MRP inhibitor MK571 (data not shown).

Once in the bloodstream, the water-soluble AL-I-NOSO ${ }_{3}$ must then be actively transported into kidney cells. OAT1, OAT2, and OAT3 are localized in the proximal tubule basolateral membrane 


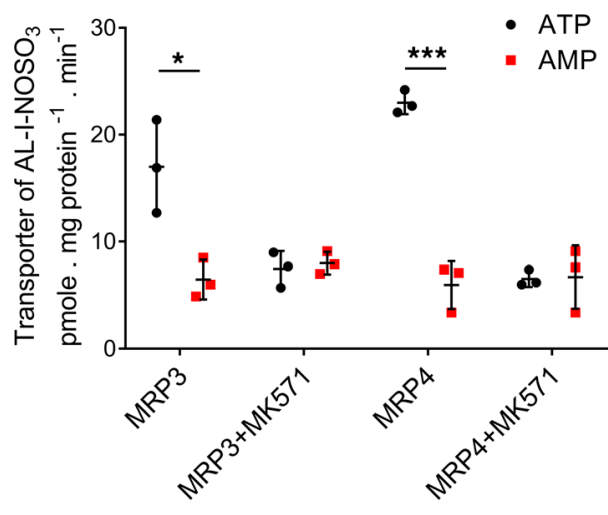

Figure 6. Membrane transport of AL-I-NOSO $3_{3}$. Uptake of $0.5 \mu \mathrm{M} \mathrm{AL-I-NOSO}$ into inside-out plasma membrane vesicles overexpressing MRP3 or MRP4 in the presence of $4 \mathrm{mM}$ ATP or $4 \mathrm{mM} \mathrm{AMP \text {, }}$ with or without $100 \mu \mathrm{M}$ MK571. $n=3$. Statistical significance was calculated using $t$ test. ${ }^{*} P<0.05$, ${ }^{* * *} P<0.001$. Quantitative results are presented as average \pm SD.

(reviewed in ref. 39), whereas OAT4 is typically expressed in the apical membrane. The lumen of kidney cells in our MPS model is oriented such that AA-I and other compounds have direct exposure to the apical membrane of the proximal tubule cells. Thus, our experimental design of the linked liver-kidney MPS involved apical exposure of kidney cells to hepatic effluent, suggesting that OAT4 is the primary uptake transporter, whereas in vivo transport is likely a combination of basolateral uptake from the blood and apical reabsorption from the glomerular filtrate (Figure 7).

Although this study has several strengths, there are also limitations to the current model. Both liver and kidney chips contain a single cell type (hepatic parenchymal cells and primary proximal tubular epithelial, respectively), whereas in vivo multiple cell types are present in each of these tissues. The two chips are connected directly via polyethylene tubing, in contrast to the in vivo situation, in which both organs have extensive, specialized vascular networks. Additionally, the perfusate we used is an artificial buffer medium that lacks proteins and other biological signaling molecules present in the bloodstream. However, these limitations, and more, are present in traditional 2D cell culture, and our data provide additional evidence that the $3 \mathrm{~S}$, microfluidic architecture of the Nortis device has substantial advantages over traditional cell culture techniques.

In conclusion, the liver appears to play a significant role in the activation of AA-I, which clinically results in progressive kidney failure and concomitant uroepithelial carcinogenesis. AL adducts of proteins and/or DNA in human kidney proximal tubule cells were found when AA-I was first metabolized by human liver cells. These data suggest that hepatic NQO1 and SULT work in concert to form the highly nephrotoxic metabolite, AL-I-NOSO ${ }_{3}$, which is then actively transported out of the liver via MRP transporters and then taken into the kidney via OATs.

Toxicity testing is central to the evaluation of risks from drugs and environmental exposures and serves as the basis for many public health and regulatory decisions. Strategies such as the Tox21 Initiative (40) are designed to improve the predictability of adverse outcomes and to reduce the use of animals in systems-level analysis in preclinical pharmacology and toxicology. Recent efforts have focused on developing in vitro models, such as in silico PK/PD modeling, high-throughput screening for nuclear receptor signaling and stress pathway assays, and adverse outcome pathway-based toxicity modeling (41). Finally, to our knowledge, this is the first example of microfluidically linked human organs-on-chips being applied to this type of investigation. While the ultimate role in organ-on-chips in human toxicology remains to be elucidated, such systems complement studies using animal models and, ultimately, may prove superior in predicting cellular response to injury in humans.

\section{Methods}

\section{Animals}

Adult male Sprague-Dawley rats, 40-60 days old, were used to obtain kidney and rat liver tissues (Charles River). Rat PTECs were isolated from rat kidneys using in vitro collagenase digestion. Primary rat hepatocytes were isolated by two-step collagenase digestion following isolation.

\section{Isolation and culturing of PTECs in MPS chips}

Human PTECs were isolated from surgically dissected kidney tissues from human donors (Him 20, Him 23, Him 25, Bio 13, and Bio 26; demographic information of PTECs donors is listed in Supplemental Table 1) using a protocol similar to that used for isolating rat PTECs. Isolated PTECs were grown in a 


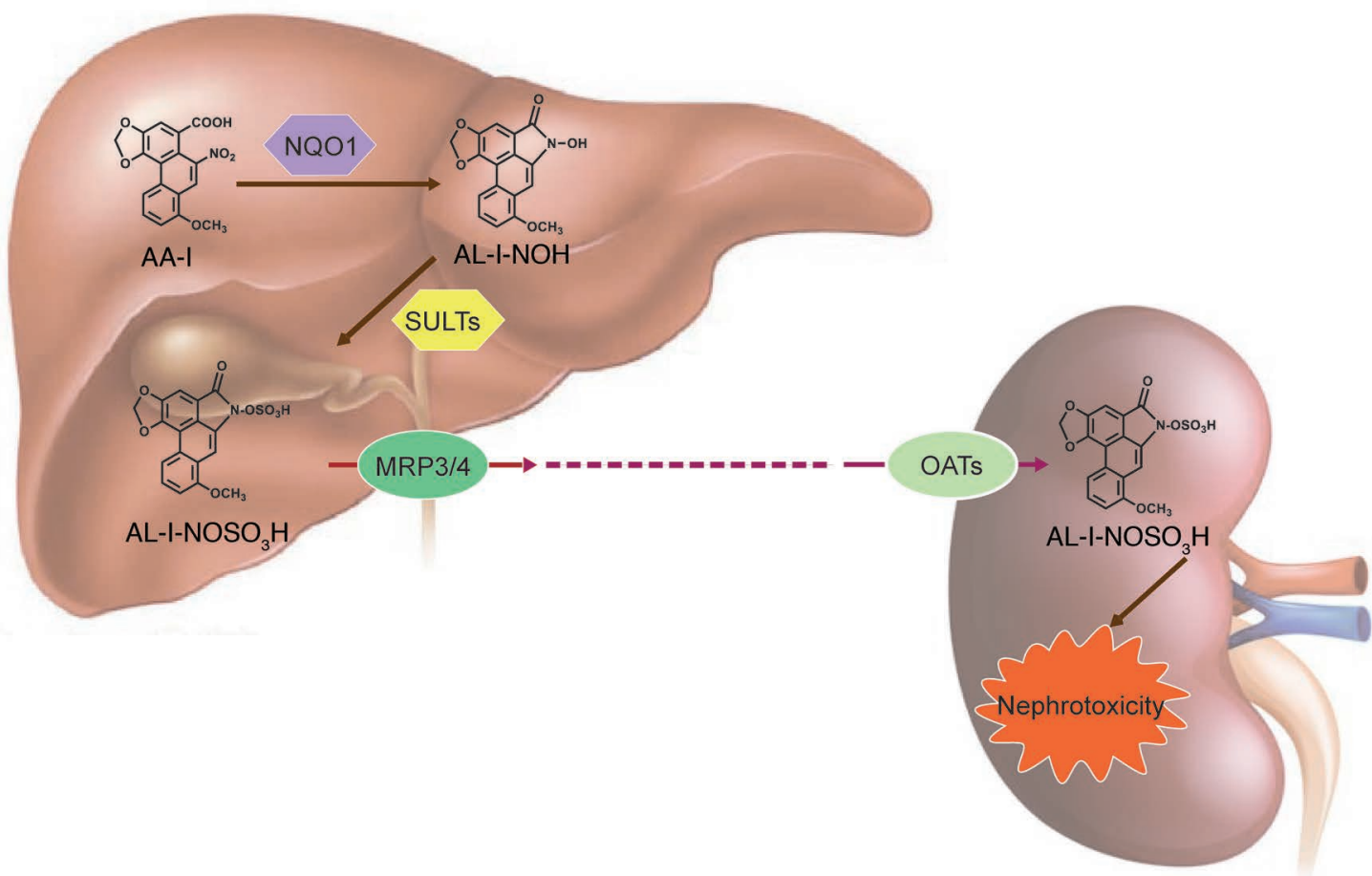

Figure 7. AA-I metabolic pathway illustrating the interaction of liver and kidney. The sulfate-conjugated metabolite of $A A-I$ and $A L-I-N O H, A L-I-N O-$ $\mathrm{SO}_{3}$, is formed by hepatic enzymes, including reduction via NQO1, and conjugation with SULTs. AL-I-NOSO is then actively transported out of liver potentially via MRP3/4 and then taken into kidney via the OAT transporters. AL-I-NOSO forms DNA and protein adducts, leading to nephrotoxicity. (Liver and kidney may also produce nontoxic metabolites of AA-I, specifically AA-la and AL-I, and their conjugates.)

2D flask with PTEC culture media, including MEM/F12 media supplemented with $2.5 \mathrm{mM}$ glutamine, 1:100 diluted ITSA, $50 \mathrm{nM}$ hydrocortisone, and 1:100 diluted Antibiotic-Antimycotic (Gibco). After expanding cells in a 2D monolayer culture, PTECs were resuspended using $0.01 \%$ trypsin and then injected into Nortis MPS chips filled with collagen type I (Figure 1A). After cell injection and stabilization overnight in a $37^{\circ} \mathrm{C}, 5 \% \mathrm{CO}_{2}$ incubator, the MPS chips were connected with C-Flex tubing, and flow was initiated with PTEC culture media at a flow rate of $0.5 \mu 1 / \mathrm{min}$, controlled by infusion syringe pumps (KD Scientific Inc., model KDS220). PTECs in MPS chips can be maintained for more than 2 weeks prior to experimental treatments.

\section{Source of hepatocytes and hepatocyte culture in MPS chips}

Freshly isolated human hepatocytes or cryopreserved human hepatocytes were purchased from Triangle Research Labs (HUM4096A and HUM4097B) or were received from the Liver Tissue Cell Distribution System (NIH service; donor 15-002) (demographic information of hepatocytes donors is listed in Supplemental Table 1; Supplemental Table 2 lists the specific human liver and kidney cell preparations used in each of the various experiments). All MPS chips were precoated with $0.1 \mathrm{mg} / \mathrm{ml}$ collagen type $\mathrm{I}$ in PBS with $0.1 \%$ acetic acid at $37^{\circ} \mathrm{C}, 5 \% \mathrm{CO}_{2}$ incubator, for 1 hour. For plating hepatocytes into MPS culture, $0.1-0.2 \mathrm{ml}$ of a cell suspension at a density of $2 \times 10^{6}$ hepatocytes $/ \mathrm{ml}$ was injected into each chip via the abluminal ports. William's E media supplemented with 5\% FBS, $100 \mathrm{mg} / \mathrm{ml}$ penicillin streptomycin, $100 \mathrm{nM}$ dexamethasone, $1 \mu \mathrm{g} / \mathrm{ml}$ Gibco Fungizone in a 1:100 diluted of ITS $^{+}$and 0.2 mM glutaMAX was used for the hepatocyte plating media. After 4 hours of plating, the hepatocytes were overlaid with Matrigel (Corning, $0.23 \mathrm{mg} / \mathrm{ml}$ ) in maintenance media (plating media but without FBS) via abluminal ports. Following maintenance of cells at $37^{\circ} \mathrm{C}, 5 \% \mathrm{CO}_{2}$ incubator overnight, cells in MPS chips were held at $37^{\circ} \mathrm{C}$ with $5 \% \mathrm{CO}_{2}$ in a sterile incubator, and were maintained in maintenance media with abluminal flow at flow rates between 5 and $10 \mu \mathrm{l} / \mathrm{h}$ via infusion/syringe pumps (KD Scientific Inc., model KDS220). Hepatocytes in MPS chips were maintained with high viability for up to 5-7 days prior to further treatment. 


\section{AA-I treatment in cells cultured in MPS}

AA-I and dicumarol were purchased from MilliporeSigma (catalog A5512 and M1390). AL-I-NOSO 3 and AL-I-NOH were synthesized as described previously (42). Probenecid (water soluble) was purchased from ThermoFisher Scientific (catalog P36400).

PTECs cultured in MPS were checked for cell confluency and tubule morphology before AA-I treatment. Only kidney MPS with $95 \%-100 \%$ confluence were used for further treatment. Two groups of cells cultured in MPS were subjected to AA-I treatment: (a) a kidney-only group that has only 3D PTECs cultured in MPS chips and (b) a liver $\rightarrow$ kidney group that has MPS chips with 3D cultured hepatocytes connected upstream of another MPS chip with PTECs. The MPS model connecting liver and kidney utilized C-Flex tubing (Figure 1C, flow is from abluminal liver chip into luminal kidney chip). Cells cultured in the MPS were treated with AA-I or AL-I-NOSO ${ }_{3}$, with and without dicumarol or probenecid in the treatment media (mixtures of PTEC culture media) and hepatocytes in maintenance media utilized a volume ratio of 50:50 for 24 hours at a flow rate of $1.5 \mu \mathrm{l} / \mathrm{min}$. After AA-I treatment, LIVE/DEAD staining was used to analyze AA-I-mediated cell death in hepatocytes and PTECs (shown in Supplemental Figure 1). Quantitative percentage cytotoxicity was calculated from the ratio of dead cells (red fluorescent-stained cells from LIVE/DEAD staining) and detached cells to the total cell number (Hoechst 33342-stained cells) (Figure 2D, $n=5$ ). Effluents were collected and stored in $-80^{\circ} \mathrm{C}$ for later analysis of organ-specific injury biomarkers.

\section{LIVE/DEAD staining}

The LIVE/DEAD viability/cytotoxicity kit and Hoechst 33342 (Life Technologies) were used to distinguish viable cells from dead cells according to the manufacturer's specifications. Briefly, calcein AM (final concentration $2 \mu \mathrm{M}$ ), EthD-1 (final concentration $4 \mu \mathrm{M}$ ), and Hoechst 33342 (final concentration $0.1 \mu \mathrm{g}$ / $\mathrm{ml}$ ) were diluted in prewarmed D-PBS. MPS chips were perfused at $5 \mu \mathrm{l} / \mathrm{min}$ via a lumenal port of kidney MPS for 20 minutes and then incubated for 10 minutes at $37^{\circ} \mathrm{C}$. After the staining procedure, chips were imaged using fluorescent microscopy (Nikon Eclipse Ti-S inverted microscope equipped with a spinning disk confocal apparatus, 3i-Intelligent Imaging Innovations) to calculate the numbers of dead cells (redstained cells) and Hoechst 33342-stained cells. All results were repeated for 3-4 independent experiments (biological replicates), each of which used 5-8 devices (technical replicates). Representative images from the various experiments and replicate devices are shown in Figures 2-5.

Immunocytochemistry staining for AL-I adducts in kidney MPS and NQ01 in liver MPS Rabbit monoclonal anti-AL adduct antibodies were raised against a mixture of aristolactam adducted DNA and albumin (T.A. Rosenquist et al., unpublished observations). Antibodies were purified from hybridoma cell supernatant by protein-A affinity chromatography. Prior to staining, $4 \%$ formaldehyde-fixed kidney MPS were bleached with $0.3 \% \mathrm{H}_{2} \mathrm{O}_{2}$ for 5 minutes under an antigen retrieval procedure (boiled $10 \mathrm{mM}$ Tris- $\mathrm{HCl}, \mathrm{pH} 9$, $1 \mathrm{mM}$ EDTA buffer) for 10 minutes at a flow rate of $10 \mu \mathrm{l} / \mathrm{min}$. Typical immunocytochemistry staining procedures, including blocking, primary antibody incubation, washing, and secondary antibody incubation, were performed using reagent-loaded syringes, controlled at flow rates between 5 and $10 \mu 1 / \mathrm{min}$ via infusion-syringe pumps (KD Scientific Inc., model KDS220). Tyramide SuperBoost kits (goat anti rabbit IgG ${ }^{2}$ and Alexa Fluor 647-labeled tyramide were included, ThermoFisher Scientific) were used to enhance the signal of AL adducts, following the manufacturers' protocol. Diluted ProLong Gold Antifade Mountant with DAPI reagents (ThermoFisher Scientific, catalog P-36931) was used in the final mounting. Secondary antibody-stained (without primary antibody incubation) kidney MPS were used to check for nonspecific binding (Supplemental Figure 2). Cells were imaged using fluorescent microscopy with a Nikon Eclipse Ti-S microscope. All immunocytochemistry staining results were repeated in triplicate $(n=3)$. Representative images are shown in Figures 2-5.

\section{Analysis of organ-specific injury biomarker- KIM-1 and ALT}

Meso Scale Discovery immunoassay kits (Meso Scale Diagnostic Inc.) were used to measure KIM-1 (rat and human) in effluents following the manufacturer's suggested protocol. The levels of ALT were measured by Phoenix Central Laboratory (Mukilteo).

Transport of AL-I-NOSO in OAT1/3-HEK or OAT4-COS-7 cells Cell culture. Stably transfected COS-7/OAT4 cells and parent nontransfected COS-7 cells were provided by Guofeng You of the Department of Pharmaceutics, Rutgers University, New Brunswick, New Jersey, 
USA. Cells were cultured at $37^{\circ} \mathrm{C}$ and $5 \% \mathrm{CO}_{2}$ in Dulbecco's modified Eagle's medium (Gibco) supplemented with 10\% FBS (VWR) and Antibiotic-Antimycotic (Gibco). COS-7/OAT4 cells were maintained in the presence of additional G418 at a concentration of $200 \mu \mathrm{g} / \mathrm{ml}$.

Uptake assay. For uptake measurements, HEK/OAT1 or OAT3 cells, COS-7/OAT4 cells, and mock control HEK and COS-7 cells were seeded on BioCoat collagen I-coated 24-well plates at a density of 5 $\times 10^{4}$ cells/well and cultured for 2 days. Cells were then washed once with $1 \mathrm{ml}$ HBSS (Gibco) and preincubated at $37^{\circ} \mathrm{C}$ with $0.5 \mathrm{ml}$ HBSS in the presence or absence of probenecid at a final concentration of 2 $\mathrm{mM}$ for 10 minutes. Uptake was initiated by adding $0.5 \mathrm{ml} \mathrm{HBSS}$ containing AL-I-NOSO $(0.5 \mu \mathrm{M})$ or the OAT4 model substrate estrone-3-sulfate $(1 \mu \mathrm{M})$ in the presence or absence of probenecid at a final concentration of $2 \mathrm{mM}$, and incubation was continued for 6 minutes at $37^{\circ} \mathrm{C}$. The uptake reaction was stopped by rapidly washing the cells with ice-cold HBSS. Cells were then lysed for 5 minutes with $0.5 \mathrm{ml}$ acetonitrile containing $32 \mathrm{ng} / \mathrm{ml}$ estradiol-3-sulfate. Cell extracts were centrifuged at 22,000 $\mathrm{g}$ for 5 minutes, and the supernatant was evaporated to dryness immediately and kept on dry ice. The residuals were reconstituted in $100 \mu \mathrm{m}$ methanol/water (1:1, vol/vol) immediately prior to LC-MS analysis. The estradiol-3-sulfate added in cell extracts was used as an internal standard. Cells used for BCA protein assay were solubilized with $0.2 \mathrm{ml} 0.1 \mathrm{NaOH}$. The amount of $\mathrm{AL}_{-}-\mathrm{NOSO}_{3}$ or estrone-3-sulfate in cell extracts normalized to protein content was presented as cellular uptake activity (Supplemental Figure 6).

LC/MS analysis. Chromatographic separation was achieved using a Waters Symmetry $\mathrm{C}_{18}(2.1 \times 50$ $\mathrm{mm}, 3.5 \mu \mathrm{m}$ ) column (Waters) on an Agilent $1200 \mathrm{LC}$ system. The elution was performed at a flow rate of $0.3 \mathrm{ml} / \mathrm{min}$ with the mobile phase containing methanol, acetonitrile, and $10 \mathrm{mM}$ ammonium acetate (native $\mathrm{pH}$ ) at a ratio of 30:14:56 (vol/vol/vol). Mass spectrum analysis was carried out using a negative mode electrospray ionization method on an Agilent 6410 triple quadrupole tandem mass spectrometer. The ionization and fragmentation parameters were set as follows: capillary voltage, 3,000 V; gas temperature, $350^{\circ} \mathrm{C}$; gas flow rate, $11 \mathrm{l} / \mathrm{min}$; nebulizer, 35 psi; fragmentor, $135 \mathrm{~V}$. Single ion monitoring (SIM) at $m / z 388$ was applied for detection of AL-I-NOSO (chromatograph in Supplemental Figure 7). Since isotopically labeled AL-I-NOSO 3 was not available, estradiol-3-sulfate was used as an internal standard for quantification of $\mathrm{AL}-\mathrm{I}-\mathrm{NOSO}_{3}$.

Inside-out plasma membrane vesicle transport of AL-I-NOSO Efflux of $\mathrm{AL}_{-}-\mathrm{NOSO}_{3}$ by MRP3 or MRP4 was investigated using inside-out plasma membrane vesicles isolated from HEK293 cells overexpressing MRP3 or MRP4 (MRP3-HEK-VT or MRP4-HEKVT, respectively) (SOLVO Biotechnology). ATP-dependent uptake of substrates into inside-out plasma membrane vesicles was carried out using a 96-well rapid filtration method according to manufacturer's instructions (SOLVO Biotechnology). Briefly, AL-I-NOSO $(0.5 \mu \mathrm{M})$ or the probe substrate estradiol-17- $\beta$-glucuronide $(10 \mu \mathrm{M})$ was incubated with membrane vesicles (25 $\mu$ g protein) in the presence of ATP or AMP (4 mM) in buffer solution ( $\mathrm{pH} 7.4$ ) containing $10 \mathrm{mM}$ Tris/ $\mathrm{HCl}, 10 \mathrm{mM} \mathrm{MgCl}$, and 250 $\mathrm{mM}$ sucrose at $37^{\circ} \mathrm{C}$ for 5 minutes, with or without MK571 $(100 \mu \mathrm{M})$. Transport was terminated by the addition of $175 \mu \mathrm{l}$ of an ice-cold washing buffer $(10 \mathrm{mM}$ Tris/ $\mathrm{HCl}, 250 \mathrm{mM}$ sucrose, and 0.02\% BSA, $\mathrm{pH}$ 7.4), followed by immediate rapid filtration through a Millipore 96-well glass-fiber filter plate. Each well was then washed 5 times with the ice-cold washing buffer. The compound trapped in membrane vesicles that were retained on filters was finally released by the addition of $200 \mu 1$ acetone containing internal standard estrodiol-3-sulfate $(50 \mathrm{ng} / \mathrm{ml})$. The membrane vesicle lysate was evaporated under $\mathrm{N}_{2}$ flow, and the residual was reconstituted with methanol/water (50:50, vol/vol) and immediately subject to LC/MS analysis as described above.

\section{Immunocytochemistry staining for NQO-1 in liver MPS}

Anti-NQO1 antibody (rabbit polyclonal, ab34173, Abcam) was used. Cells were fixed with 4\% formaldehyde in PBS. After antigen retrieval with warm citrate buffer (10 mM citric acid, $0.05 \%$ Tween 20, pH 6.0) for 20 minutes, samples were permeabilized with PBST (PBS plus $0.1 \%$ Tween 20) for 1 hour and blocked with $1 \%$ BSA and $10 \%$ serum in PBSG $(10 \mathrm{mg} / \mathrm{ml}$ glycine, $0.1 \%$ Tween 20$)$ for an additional 1 hour. The samples were then incubated with primary and secondary antibodies followed by washing, using a standard immunocytochemistry protocol. Secondary antibodies used were goat anti-rabbit IgG $(\mathrm{H}+\mathrm{L})$ secondary antibody, Alexa Fluor 488 conjugate (ThermoFisher Scientific). Diluted ProLong Gold Antifade Mountant with DAPI reagents (catalog P-36931, ThermoFisher Scientific) were used in 
the final step of staining. Cells were imaged using fluorescent microscopy with the Nikon Eclipse Ti-S microscope for detecting the intensity of green and blue fluorescence.

\section{Statistics}

GraphPad Prism 6 was used to plot and analyze results. All results of MPS culture were from 3-4 independent AA treatment experiments (3-4 times through experimental setup, including cell prep and MPS system setup; shown in Supplemental Table 2). The qualitative results of graphs with error bars are represented as mean (average) \pm SD. Nonparametric $t$ test and multiple $t$ test with correction for multiple comparisons were analyzed. A $P$ value of less than 0.05 was considered significant.

\section{Study approval}

Animals were maintained in Association for Assessment and Accreditation of Laboratory Animal Care International-approved vivarium facilities at the University of Washington, and all protocols for animal use and care were approved by the University of Washington Institutional Animal Care and Use Committee. Human PTECs were isolated from surgically dissected kidney tissues from human donors, following institutional IRB approval. Freshly isolated human hepatocytes or cryopreserved human hepatocytes were purchased or received following IRB protocol review and approval.

\section{Author contributions}

SYC, EJK, JH, and DLE conceived and supervised the overall project. SYC, VSS, AC, CKY, CG, QM, DS, JW, JH, DLE, and EJK designed experiments and analyzed data. SYC, EJW, AC, and CG performed experiments. SYC, VSS, QM, TAR, KGD, TN, APG, EJK, JH, and DLE wrote and edited the manuscript. DLE is the lead contact and is responsible for all aspects of the manuscript submission process as well as development of the liver-on-a-chip approach. JH and EJK shared equally in responsibilities for the grant funding that supported this work and for the oversight of the development of kidneyon-a-chip approach. VSS developed and provided access to all of the AA metabolites, antibodies, and other resources required for this study.

\section{Acknowledgments}

We acknowledge help from the Biomarker Core Facility of the National Institute of Environmental Health Sciences Center for Exposures, Diseases, Genes and Environment (P30ES07033; to QM, EJK, JH, DLE), the University of Washington Pharmacokinetics and Modeling Lab, and the University of Washington School of Pharmacy Mass Spectrometry Facility. This publication was developed under NIH grant 5UH3TR000504-04 (to JH) and Assistance Agreement 83573801 awarded by the US Environmental Protection Agency to Elaine M. Faustman, Department of Environmental and Occupational Health Sciences, University of Washington, with additional support from the Henry and Marsha Laufer Foundation to VSS, TAR, KGD and APG. It has not been formally reviewed by EPA. The views expressed in this document are solely those of the authors and do not necessarily reflect those of the agency. EPA does not endorse any products or commercial services mentioned in this publication.

Address correspondence to: David L. Eaton, Environmental and Occupational Health Sciences, University of Washington, 4225 Roosevelt Way, NE, Suite 100, Seattle, Washington 98105-6099, USA. Phone: 206.685.3785; Email: deaton@uw.edu. Or to: Viktoriya S. Sidorenko, Department of Pharmacological Sciences, Stony Brook University Medical School, BST, L8 room 147, Stony Brook, New York 11794-865, USA. Phone: 631.444.9723; Email: viktoriya.sidorenko@stonybrook.edu. Or to: Edward J. Kelly, Department of Pharmaceutics, University of Washington, 1959 NE Pacific St, HSB Rm H272, Box 357610, Seattle, Washington 98195 USA. Phone: 206.685.4641; Email: edkelly@uw.edu. Or to: Jonathan Himmelfarb, Kidney Research Institute, Division of Nephrology, Box 359606, 325 Ninth Avenue, Seattle, Washington 98104, USA. Phone: 206.616.4717; Email: Jhimmelfarb@nephrology.washington.edu.

1. Vanherweghem JL, et al. Rapidly progressive interstitial renal fibrosis in young women: association with slimming regimen including Chinese herbs. Lancet. 1993;341(8842):387-391.

2. Grollman AP, et al. Aristolochic acid and the etiology of endemic (Balkan) nephropathy. Proc Natl Acad Sci USA. 2007;104(29):12129-12134. 
3. Shibutani S, Dong H, Suzuki N, Ueda S, Miller F, Grollman AP. Selective toxicity of aristolochic acids I and II. Drug Metab Dispos. 2007;35(7):1217-1222.

4. Chen CH, et al. Aristolochic acid-associated urothelial cancer in Taiwan. Proc Natl Acad Sci USA. 2012;109(21):8241-8246.

5. Jelaković B, et al. Aristolactam-DNA adducts are a biomarker of environmental exposure to aristolochic acid. Kidney Int. 2012;81(6):559-567.

6. Sidorenko VS, et al. Bioactivation of the human carcinogen aristolochic acid. Carcinogenesis. 2014;35(8):1814-1822.

7. Hashimoto K, et al. Sulfotransferase-1A1-dependent bioactivation of aristolochic acid I and N-hydroxyaristolactam I in human cells. Carcinogenesis. 2016;37(7):647-655.

8. Bhatia SN, Ingber DE. Microfluidic organs-on-chips. Nat Biotechnol. 2014;32(8):760-772.

9. Weber EJ, et al. Development of a microphysiological model of human kidney proximal tubule function. Kidney Int. 2016;90(3):627-637.

10. Han WK, Bailly V, Abichandani R, Thadhani R, Bonventre JV. Kidney Injury Molecule-1 (KIM-1): a novel biomarker for human renal proximal tubule injury. Kidney Int. 2002;62(1):237-244.

11. Stiborová M, Martínek V, Frei E, Arlt VM, Schmeiser HH. Enzymes metabolizing aristolochic acid and their contribution to the development of aristolochic acid nephropathy and urothelial cancer. Curr Drug Metab. 2013;14(6):695-705.

12. Aleksunes LM, Goedken M, Manautou JE. Up-regulation of NAD(P)H quinone oxidoreductase 1 during human liver injury. World J Gastroenterol. 2006;12(12):1937-1940.

13. Bárta F, Levová K, Frei E, Schmeiser HH, Arlt VM, Stiborová M. The effect of aristolochic acid I on expression of NAD(P) H:quinone oxidoreductase in mice and rats--a comparative study. Mutat Res Genet Toxicol Environ Mutagen. 2014;768:1-7.

14. Arlt VM, et al. Impact of genetic modulation of SULT1A enzymes on DNA adduct formation by aristolochic acids and 3-nitrobenzanthrone. Arch Toxicol. 2017;91(4):1957-1975.

15. Meinl W, Pabel U, Osterloh-Quiroz M, Hengstler JG, Glatt H. Human sulphotransferases are involved in the activation of aristolochic acids and are expressed in renal target tissue. Int J Cancer. 2006;118(5):1090-1097.

16. Stiborová M, Mareš J, Frei E, Arlt VM, Martínek V, Schmeiser HH. The human carcinogen aristolochic acid i is activated to form DNA adducts by human $\mathrm{NAD}(\mathrm{P}) \mathrm{H}$ :quinone oxidoreductase without the contribution of acetyltransferases or sulfotransferases. Environ Mol Mutagen. 2011;52(6):448-459.

17. Xue X, et al. Critical role of organic anion transporters 1 and 3 in kidney accumulation and toxicity of aristolochic acid I. Mol Pharm. 2011;8(6):2183-2192.

18. Bakhiya N, Arlt VM, Bahn A, Burckhardt G, Phillips DH, Glatt H. Molecular evidence for an involvement of organic anion transporters (OATs) in aristolochic acid nephropathy. Toxicology. 2009;264(1-2):74-79.

19. Ekaratanawong $\mathrm{S}$, et al. Human organic anion transporter 4 is a renal apical organic anion/dicarboxylate exchanger in the proximal tubules. J Pharmacol Sci. 2004;94(3):297-304.

20. Rosenquist TA. Genetic loci that affect aristolochic acid-induced nephrotoxicity in the mouse. Am J Physiol Renal Physiol. 2011;300(6):F1360-F1367.

21. National Toxicology Program. NTP 12th Report on Carcinogens. Rep Carcinog. 2011;12:iii-499.

22. Sato N, et al. Acute nephrotoxicity of aristolochic acids in mice. J Pharm Pharmacol. 2004;56(2):221-229.

23. Hsieh SC, Lin IH, Tseng WL, Lee CH, Wang JD. Prescription profile of potentially aristolochic acid containing Chinese herbal products: an analysis of National Health Insurance data in Taiwan between 1997 and 2003. Chin Med. 2008;3:13.

24. Vanherweghem LJ. Misuse of herbal remedies: the case of an outbreak of terminal renal failure in Belgium (Chinese herbs nephropathy). J Altern Complement Med. 1998;4(1):9-13.

25. Priestap HA, et al. Aristolochic acid I metabolism in the isolated perfused rat kidney. Chem Res Toxicol. 2012;25(1):130-139.

26. Ozer J, Ratner M, Shaw M, Bailey W, Schomaker S. The current state of serum biomarkers of hepatotoxicity. Toxicology. 2008;245(3):194-205.

27. Lind C, Cadenas E, Hochstein P, Ernster L. DT-diaphorase: purification, properties, and function. Meth Enzymol. 1990;186:287-301

28. Chen M, et al. Inhibition of renal NQO1 activity by dicoumarol suppresses nitroreduction of aristolochic acid I and attenuates its nephrotoxicity. Toxicol Sci. 2011;122(2):288-296.

29. Stiborová M, et al. The influence of dicoumarol on the bioactivation of the carcinogen aristolochic acid I in rats. Mutagenesis. 2014;29(3):189-200.

30. Pfau W, Pool-Zobel BL, von der Lieth CW, Wiessler M. The structural basis for the mutagenicity of aristolochic acid. Cancer Lett. 1990;55(1):7-11.

31. Riches Z, Stanley EL, Bloomer JC, Coughtrie MW. Quantitative evaluation of the expression and activity of five major sulfotransferases (SULTs) in human tissues: the SULT "pie". Drug Metab Dispos. 2009;37(11):2255-2261.

32. Krumbiegel G, Hallensleben J, Mennicke WH, Rittmann N, Roth HJ. Studies on the metabolism of aristolochic acids I and II Xenobiotica. 1987;17(8):981-991.

33. Glatt H. Sulfation and sulfotransferases 4: bioactivation of mutagens via sulfation. FASEB J. 1997;11(5):314-321.

34. Gamage N, et al. Human sulfotransferases and their role in chemical metabolism. Toxicol Sci. 2006;90(1):5-22.

35. Skipper PL, Obiedzinski MW, Tannenbaum SR, Miller DW, Mitchum RK, Kadlubar FF. Identification of the major serum albumin adduct formed by 4-aminobiphenyl in vivo in rats. Cancer Res. 1985;45(10):5122-5127.

36. Ma L, Glatt HR. Albumin strongly prolongs the lifetime of chemically reactive sulphuric acid esters and affects their biological activities in the rat. Nova Acta Leopoldina. 2003;329:265-272.

37. Bendadani C, et al. Determination of sulfotransferase forms involved in the metabolic activation of the genotoxicant 1-hydroxymethylpyrene using bacterially expressed enzymes and genetically modified mouse models. Chem Res Toxicol. 2014;27(6):1060-1069.

38. Slot AJ, Molinski SV, Cole SP. Mammalian multidrug-resistance proteins (MRPs). Essays Biochem. 2011;50(1):179-207.

39. Hagos Y, Wolff NA. Assessment of the role of renal organic anion transporters in drug-induced nephrotoxicity. Toxins (Basel). 2010;2(8):2055-2082.

40. Knudsen TB, et al. FutureTox II: in vitro data and in silico models for predictive toxicology. Toxicol Sci. 2015;143(2):256-267.

41. Wittwehr C, et al. How adverse outcome pathways can aid the development and use of computational prediction models for regulatory toxicology. Toxicol Sci. 2017;155(2):326-336. 
42. Attaluri S, Iden CR, Bonala RR, Johnson F. Total synthesis of the aristolochic acids, their major metabolites, and related compounds. Chem Res Toxicol. 2014;27(7):1236-1242.

43. Chang SY, et al. Characterization of rat or human hepatocytes cultured in microphysiological systems (MPS) to identify hepatotoxicity. Toxicol In Vitro. 2017;40:170-183. 